\title{
CONTINUOUS
}

\section{SUBCUTANEOUS INSULIN INFUSION}

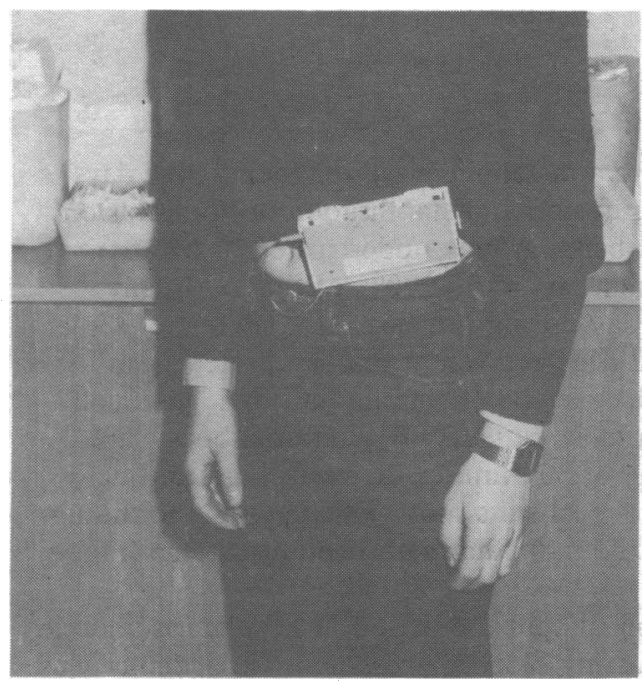

\section{Technique}

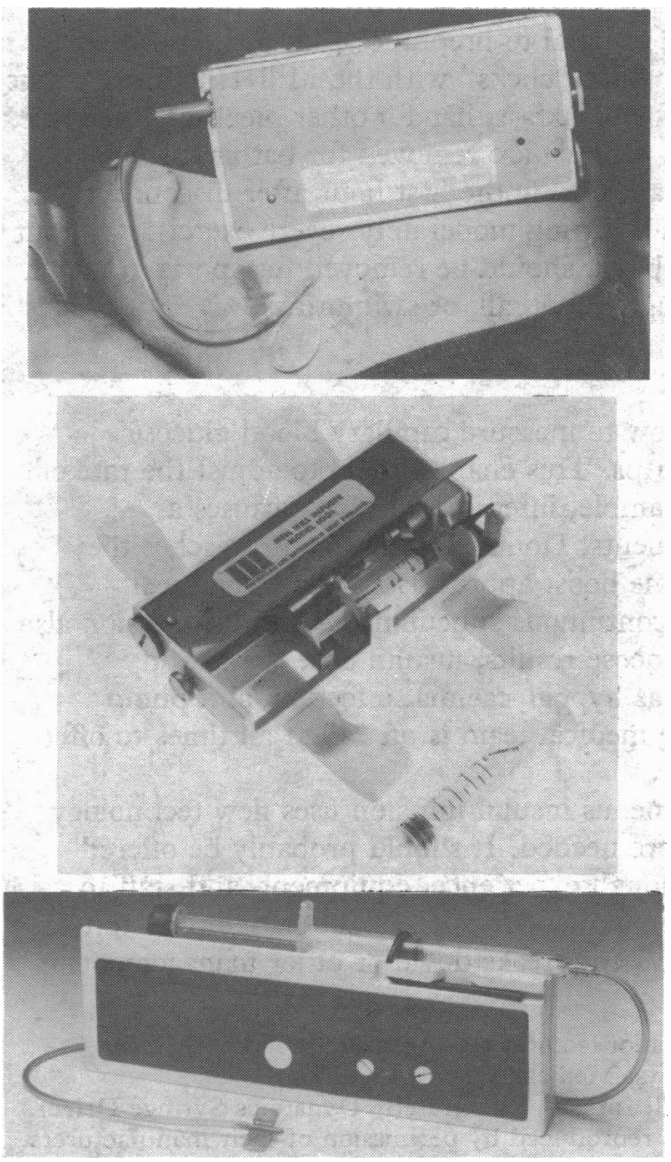

Continuous subcutaneous insulin infusion (CSII) provides a means of achieving optimal diabetic control. It is an important research tool and clinically may be particularly valuable when "super" control is essential and has not been achieved by conventional injection methods. Indications for using this technique when traditional insulin treatment has failed are pregnancy, painful diabetic neuritis, and unstable diabetes causing disabling hypoglycaemic episodes. Almost physiological control can be achieved in most cases, but there are notable exceptions, including some very unstable (brittle) diabetics who are not always improved by continuous subcutaneous insulin infusion. Continuous intramuscular insulin infusion is an experimental technique which has achieved some short-term success in brittle diabetics; continuous intravenous infusion has also been described over long periods, but the potential hazards of infection and thrombosis have restricted its general application.

The long-term, more routine use of continuous subcutaneous insulin infusion is still uncertain and will depend partly on the development of smaller, reliable pumps. Prevention or retardation of diabetic complications is a goal which all doctors seek to achieve: research into treatment with infusion pumps is very active.

\section{Delivery cannulae and implantation site}

A fine-gauge (25-G) metal needle, with wings, attached to a connecting tube (Butterfly, Abbott Laboratories) is usually used. This can be inserted by the patient, is relatively non-kinking, and is easily secured to the skin by the wings. Accidental withdrawal almost never occurs.

The cannula is reimplanted every day at a different site in the subcutaneous tissue of the anterior abdominal wall, usually at the same time as the pump insulin syringe is refilled.

\section{Insulin}

Short-acting insulin (neutral soluble purified) is used in the pump syringe. For single basal rate infusers (such as the Mill Hill $1001 \mathrm{HM}$ ) the insulin must be diluted to deliver a dose rate appropriate to requirements. Isotonic saline or the insulin diluent supplied by the insulin manufacturer is used. Current infusion pumps deliver about $2.5 \mathrm{ml}$ of insulin per day.

\section{Pumps}

Several suitable infusion pumps are available, and improved ones are being developed. Most present pumps deliver insulin from a syringe. They infuse insulin at a basal rate (the volumetric rate of which either is fixed or can be preset) and have facilities for delivering a preprandial boost either manually or electronically. The concentration of insulin in the syringe is obviously important in determining the basal infusion rate.

The pump is usually worn in a pouch, attached to a belt around the waist or a shoulder harness.

The following pumps are most readily available in the UK.

The Mill Hill Infuser, Model 1001 HM (Muirhead Medical Ltd, 19 Eldon Park, London SE25), has a fixed basal delivery rate of $66 \mu \mathrm{l} / \mathrm{h}$; a lead screw can be turned manually and the syringe plunger advanced by a 


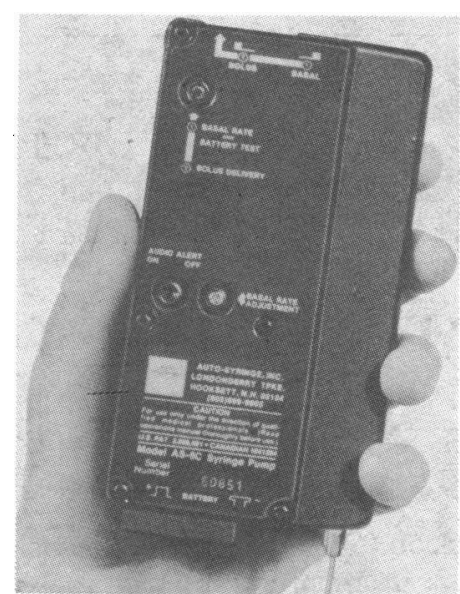

\section{Infusion strategy}

Start:

$1 / 2$ usual daily dose as basal infusion over 24 hours

$1 / 2$ usual daily dose divided equally and given as boost $15-45$ minutes betore the 3 main meals of the day

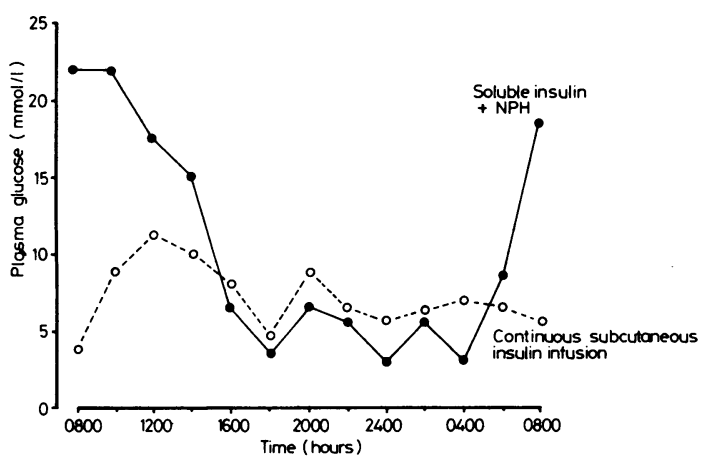

\section{Outpatient management}

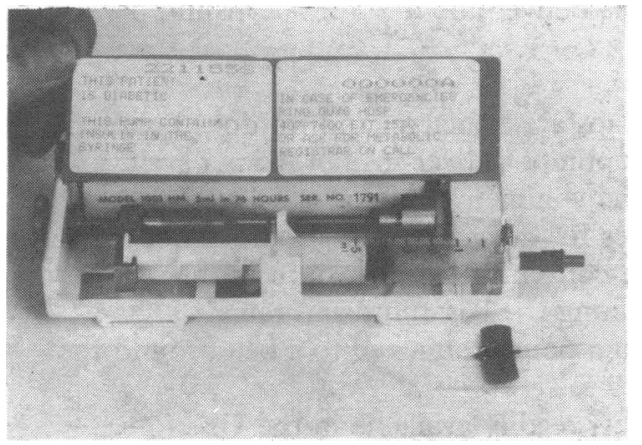

knob on the outside of the pump. This gives a measured bolus for meal times, selected by the number of countable clicks (one click $=$ one half turn of the lead screw $=53 \mu \mathrm{l}$ ).

The Graseby Dynamics Syringe Driver, Model MS26 (Graseby Dynamics, Park Avenue, Bushey, Watford, Herts), has a variable delivery rate $(0-99 \mathrm{~mm} / \mathrm{h})$, the volume of which varies according to the size of syringe used. There is an electronic boost for preprandial insulin which delivers $0.23 \mathrm{~mm}$ at each press of a button.

The Auto-Syringe Pump (C F Bond, Parkhead House, 26 Carver Street, Sheffield, latest model AS6C) has a basal rate of 25-125 $\mu \mathrm{l} / \mathrm{h}$ and electronic bolus delivery for meals with automatic reinstatement of basal rate.

Patients are usually admitted to a metabolic ward for four to five days for education about continuous infusion and stabilisation on the infusion. Assuming that the patient is not being grossly undertreated or overtreated by conventional injection treatment, about $80-100 \%$ of the total daily insulin dose will be needed per day during infusion. On day one of the admission about half of the total dose is given at the basal rate (mean value for adult diabetics 1 unit/h) and the remaining half divided equally among the three main meals. In most patients no additional boosts are given for snacks. We find a delay of 30 minutes between the mealtime insulin boost and the start of the meal suits most patients, but the timing sometimes has to be tailored to the individual's absorption pattern (range 15-45 minutes).

Blood glucose is monitored throughout the period in hospital as a guide to adjusting insulin rates. The fasting blood glucose is used particularly as a measure of the basal infusion rate and the postprandial value (about 90 minutes after a meal) as a guide to the magnitude of the mealtime boost. During the rest of the stay in hospital blood glucose control is optimised fine-tuning the basal rate and the magnitude and timing of preprandial boosts. There is no fixed ratio of basal to preprandial insulin dose; the average mealtime insulin dose is six "clicks" with the Mill Hill Infuser, but more insulin is often needed for breakfast than for other meals of equal size.

The pump and the infusion cannula are removed for bathing, the blood glucose remaining fairly constant during the first hour after disconnection. The pump may be kept in place during moderately severe exercise, without a change in the infusion rate, but it should be removed for sports in which it may be damaged, such as rugby, football, or swimming.

Patients should be taught how to measure capillary blood glucose concentrations with reagent strips. This enables them to adjust the rate of infusion at home when, for example, intercurrent illness causes a temporary increase in requirements. Home monitoring also teaches the diabetic the size of the mealtime boost appropriate for a given meal.

In our practice patients on continuous subcutaneous insulin infusion also record each day their blood glucose results, insulin dose (basal and preprandial), and events such as hypoglycaemia, infection, and pump malfunction. A member of the medical team is on call at all times to offer advice and help.

Because continuous subcutaneous insulin infusion uses new technology special skills and precautions are needed. It should probably be offered only by centres which have enough experience, equipment, and staff to provide the necessary education and supervision of the patient and to take rapid corrective action if the system breaks down or other management problems occur.

Dr J C Pickup, BM, DPHIL, is senior lecturer grade research fellow, Unit for Metabolic Medicine, Guy's Hospital Medical School, London.

The illustrations of the Mill Hill Infuser, the Graseby Dynamics Syringe Driver, and the Auto-Syringe Pump were reproduced by permission of their manufacturers. 